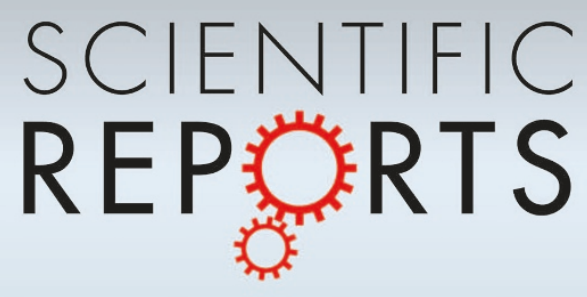

OPEN

SUBJECT AREAS:

QUANTUM OPTICS

QUANTUM DOTS

QUANTUM INFORMATION

NANOWIRES

Received

17 May 2013

Accepted

19 June 2013

Published

5 July 2013

Correspondence and requests for materials should be addressed to Y.H.C. (yhc@kaist.ac.kr)

\title{
Ultrafast single photon emitting quantum photonic structures based on a nano-obelisk
}

\author{
Je-Hyung Kim, Young-Ho Ko, Su-Hyun Gong, Suk-Min Ko \& Yong-Hoon Cho
}

Department of Physics, Graduate School of Nanoscience \& Technology (WCU), and KAIST Institute for the NanoCentury, KAIST, Daejeon, 305-701, Republic of Korea.

A key issue in a single photon source is fast and efficient generation of a single photon flux with high light extraction efficiency. Significant progress toward high-efficiency single photon sources has been demonstrated by semiconductor quantum dots, especially using narrow bandgap materials. Meanwhile, there are many obstacles, which restrict the use of wide bandgap semiconductor quantum dots as practical single photon sources in ultraviolet-visible region, despite offering free space communication and miniaturized quantum information circuits. Here we demonstrate a single InGaN quantum dot embedded in an obelisk-shaped GaN nanostructure. The nano-obelisk plays an important role in eliminating dislocations, increasing light extraction, and minimizing a built-in electric field. Based on the nano-obelisks, we observed nonconventional narrow quantum dot emission and positive biexciton binding energy, which are signatures of negligible built-in field in single InGaN quantum dots. This results in efficient and ultrafast single photon generation in the violet color region.

\footnotetext{
A single photon source that generates non-classical light provides a fundamental element for advanced information processing ${ }^{1}$, i.e., quantum information. Semiconductor quantum dots (QDs) are the most promising candidate for single photon sources due to their stability, fast generation rate, wavelength tunability, and the possibility of electrical operation ${ }^{2-4}$. In the past decade, there have been several demonstrations of semiconductor QDs embedded in microcavities ${ }^{2,5}$ such as microdisks, micropilllars, and photonic crystals, which have a high quality factor and a small mode volume. By integrating a single emitter with an optical cavity, it is possible to control light-matter interaction and enhance the radiative recombination rate and light extraction efficiency. However, difficulties in spatial and spectral matching between single QDs and the microcavity system still remain as large obstacles. Recently, instead of employing microcavities, a few groups have demonstrated highly efficient single photon sources using single emitters embedded in photonic nanostructures ${ }^{6-9}$. The photonic nanostructures could guide single photons from the emitter to the fundamental waveguide mode of the nanostructure, enhancing the spontaneous recombination rate of emitters and efficiently extracting single photons through the tapered tip of the nanostructure. Likewise, various approaches have been actively explored in long wavelength region based on narrow-bandgap semiconductors, such as $\operatorname{InAs}{ }^{10}$ (near infra-red), $\operatorname{In} \mathrm{P}^{11}$ (red), and $\mathrm{CdSe}^{12}$ (green). However, a high-efficiency single photon source based on wide-bandgap semiconductors, which emit short wavelength, is still challenging. At long wavelength, single photons should be controlled in optical fibers due to large loss in air, whereas ultraviolet (UV)-visible single photon sources have benefits in free space communication ${ }^{13}$ and miniaturized quantum information circuits with available high-sensitivity and highspeed detectors ${ }^{14,15}$. Frequency conversion of short wavelength single photons to long wavelength frequency is also possible ${ }^{16}$. The group III-nitrides are attractive materials for ultraviolet-visible optical devices and exhibit several advantages such as wide spectral tunability, large exciton binding energy ${ }^{17}$, slow surface recombination velocity $^{18}$, and strong mechanical and chemical stability, leading the evolution of solid-state lighting ${ }^{19}$. A highefficiency single photon source operating at high temperature based on III-nitride QDs can thus be anticipated. A single photon source from self-assembled GaN QDs grown by Stranski-Kranstanov mode has been demonstrated up to $200 \mathrm{~K}^{20}$. However, as a deterministic quantum light source, conventional III-nitride self-assembled QDs have some disadvantages: QDs are randomly distributed and embedded in a planar matrix, resulting in poor light extraction and difficulties to obtain single QDs. A large number of dislocations and the existence of a huge built-in electric field of a few $\mathrm{MV} / \mathrm{cm}$ in the conventional III-nitride QDs strengthen the nonradiative process and weaken the radiative recombination process ${ }^{21}$. Moreover, unlike narrow bandgap semiconductors, it is difficult to employ
} 
microcavities or nanostructures in QDs because there are no proper etchants for chemical wet etching ${ }^{22}$, and physical etching methods generally induce severe etching damage and seriously deteriorate optical properties. ${ }^{23}$ These problems fundamentally limit the practical use of nitride based QDs as a single photon source. For these reasons, although group III-nitride QDs have been extensively studied $^{21,24-26}$, there have only been a few reports on single photon generation based on III-nitride QDs ${ }^{20,27-29}$.

Here we demonstrate obelisk-shaped, high-quality nanostructures and report efficient and ultrafast single photon generation from a single InGaN QD embedded in a nano-obelisk. Obelisk-shaped nanostructures were formed by a chemical vapor-phase etching process that was recently developed by our group ${ }^{30}$. The nano-obelisk with a pyramidal tip efficiently increases light extraction, reduces the built-in electric field, and has a high crystal quality. With this unique structure, we observed linearly polarized narrow QD emissions and successfully generated efficient and ultrafast single photons, which cannot be achieved with conventional self-assembled QDs.

\section{Results}

Fabrication of nano-obelisks using chemical vapor-phase etching and epitaxial regrowth process. The formation of high-quality nanostructures by top-down etching is a challenging task in group III-nitride semiconductors due to the strong chemical and mechanical stability of III-nitride materials. To acquire a highquality quantum emitter embedded in photonic nanostructures, we followed two steps. First, we prepared a $2 \mu \mathrm{m}$-thick GaN film on a sapphire substrate, and then the GaN film was exposed to vaporphase $\mathrm{HCl}$ at $1000^{\circ} \mathrm{C} . \mathrm{HCl}$ gas efficiently activates the dissociation process in $\mathrm{GaN}$ at high temperature and induces facet- and defectselective etching. In contrast to physical etching, the chemical vaporphase etching improves the structural and optical properties of $\mathrm{GaN}$ by reducing dislocations and residual strain, and enhancing light extraction efficiency (Supplementary Sections I and II). Details of the chemical vapor-phase etching process and characterization were previously reported ${ }^{30}$. Next, based on this etched GaN nanostructure template, we regrew InGaN active layers and $\mathrm{GaN}$ capping layers using metal organic chemical vapor deposition (MOCVD). During the regrowth process, radial growth occurs more dominantly than axial growth. Figure 1a shows scanning electron microscope (SEM) images of as-formed nano-obelisks with an average diameter of $200 \mathrm{~nm}$, a height of $1.5 \mu \mathrm{m}$, and a density of $2 \sim 3 \times 10^{8} \mathrm{~cm}^{-2}$. Highly-faceted hexagonal nano-obelisks consist of a vertical column part and a pyramidal tip part; this is a favorable shape for emitting photons $^{8,9}$ and reducing a built-in electric field ${ }^{31-33}$. In the case of conventional self-assembled QDs embedded in a planar structure, a large built-in electric field reduces the oscillator strength of excitons in QDs and most emitted photons cannot escape outwards due to total internal reflection (Fig. 1b). We simulated the light extraction efficiency in the obelisk-shaped photonic nanostructures and compared it with that of nanostructures without tapered tips (i.e., pillar type) and planar substrates. The results show that the light extraction efficiency is only $4 \%$ for an emitter located in the planar structure, and it increased to $20 \%$ for the pillar type nanostructures. For an emitter embedded in the nano-obelisks, light extraction efficiency of around $36 \%$ is observed (Supplementary Section II).

Formation of quantum structures in nano-obelisks and characterization. The formation of InGaN quantum structures in nano-obelisks and their structural properties were studied using high-resolution transmission electron microscopy (HRTEM) and scanning TEM (STEM) in high-angle annular dark field (HAADF) mode, as shown in Fig. 2 . The pyramidal tip part of the nano-obelisk has $\{11 \overline{2} 2\}$ semipolar facets and the vertical column part of the nanoobelisk shows nearly nonpolar facets with an angle of about $85^{\circ}$ to the $c$-plane. In TEM images, as-formed nano-obelisks show a high-crystal
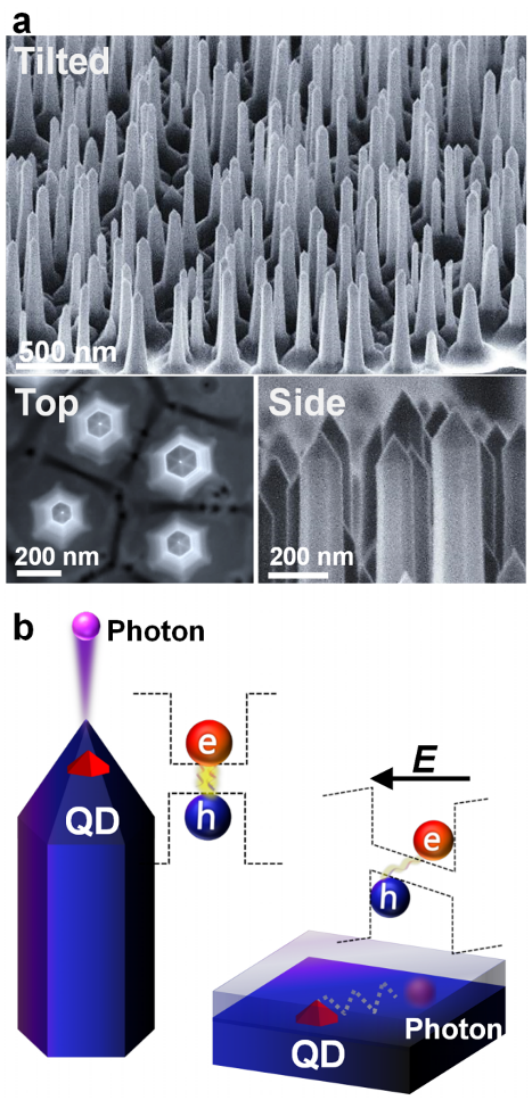

Figure 1 Formation of GaN nano-obelisks including InGaN quantum structures. (a) Tilted-, top-, and side-view SEM images for nano-obelisks. (b) Schematic representation of a QD in a nano-obelisk (left) and a conventional QD in a planar structure (right). The nano-obelisk emits photons efficiently and reduces the built-in electric field, while the planar structure captures photons inside of it and has a large built-in electric field, restricting electron-hole recombination.

quality without any dislocations, since the chemical vapor-phase etching eliminates dislocations ${ }^{30}$ and the regrowth process does not induce additional dislocations (Supplementary Section I). To understand the formation mechanism of InGaN quantum structures, we studied nano-obelisks including multiple-InGaN layers with different thicknesses. The cross-sectional TEM images of the nano-obelisk show that the InGaN quantum wells (QWs) cover the $\mathrm{GaN}$ nano-obelisk, resulting in a $\mathrm{GaN} / \mathrm{InGaN}$ core-shell structure (Fig. 2a-d). Generally, the growth rate of materials depends on their grown facets having a different surface energy, and this causes a change in the thickness of the QWs at the pyramidal facets $(0.5 \mathrm{~nm}$, $1.9 \mathrm{~nm}$, and $7 \mathrm{~nm})$ compared to that at the column facets $(1.5 \mathrm{~nm}$, $5 \mathrm{~nm}$, and $17 \mathrm{~nm}$ ) (Fig. 2c). We also note that an island is formed at the top of the pyramidal tip (Fig. 2b) (see Supplementary Sections III and IV for further analysis). Some characteristics of low-dimensional quantum structures have also been observed at the apexes or edges of micro-pyramidal structures ${ }^{34-36}$. Based on these experimental observations, we grew a single and ultrathin InGaN layer in a nano-obelisk to achieve a small size island (i.e., QD) at the top of the nano-obelisk (Fig. 2e-i), and we anticipate that a well-isolated QD with a height of around $1 \sim 2 \mathrm{~nm}$ would be formed at the top of a nano-obelisk (Fig. 2i). For the nano-obelisk with a ultrathin single QW, it is difficult to observe a clear cross-sectional TEM image of the InGaN quantum structures embedded in a nanometer-scale pyramidal tip (Fig. 2f), but we confirmed the formation of a $1 \mathrm{~nm}$ thick thin QW at the column part (Fig. 2g) and at the bottom of a nano-obelisk (Fig. 2h). 
b

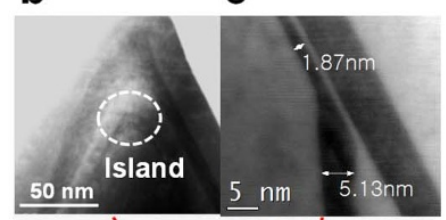

a

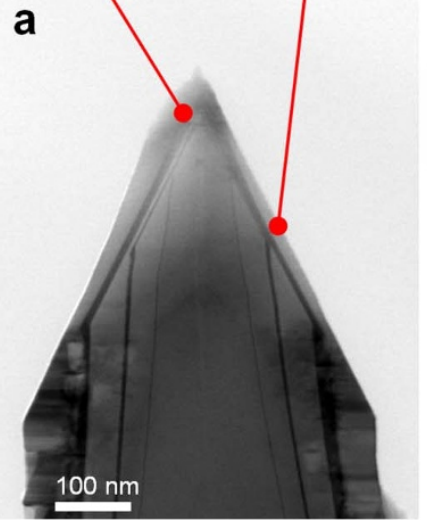

d

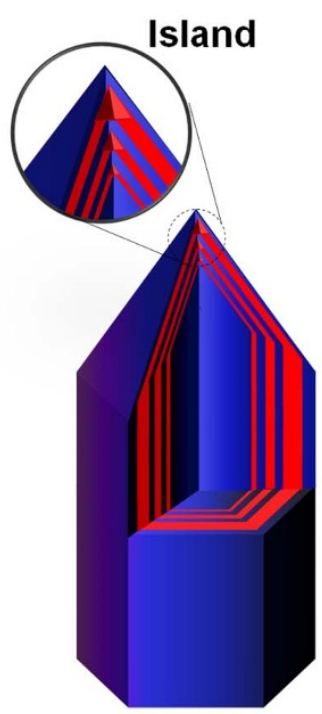

e

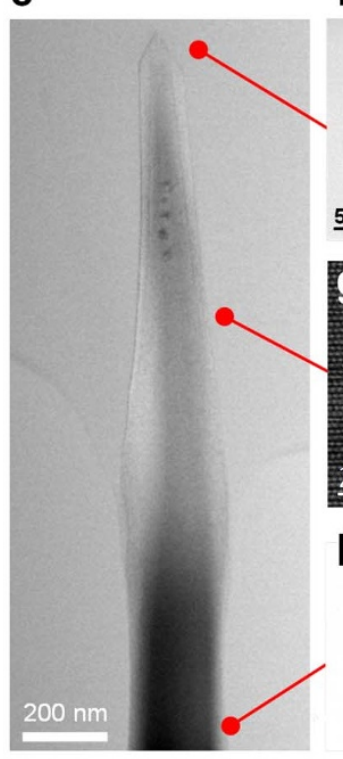

f
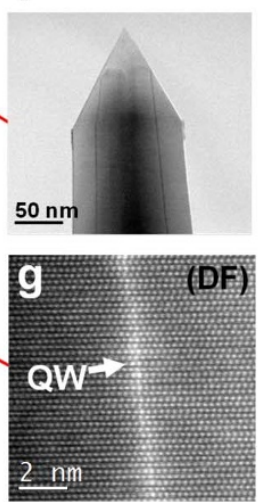

h

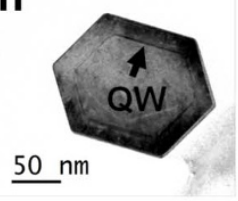

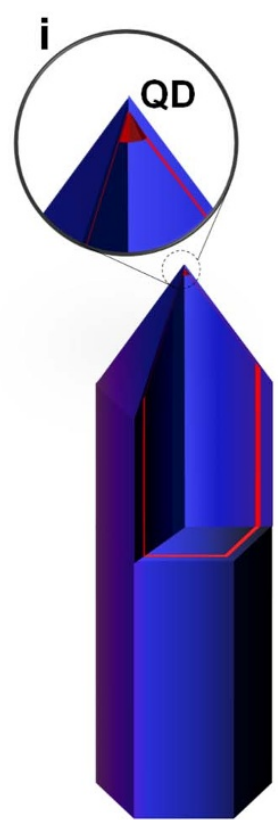

Figure 2 Formation of GaN nano-obelisks including InGaN quantum structures. (a-c) Cross-sectional TEM images for a nano-obelisk including multiple-QWs with different QW thicknesses. (b) Formation of an island at the top of a nano-obelisk. (c) QW thickness change between a pyramidal facet and a column facet. (d) Schematic illustration of a nano-obelisk including multiple QWs and an InGaN island at the top. (e-g) Cross-sectional TEM images for a nano-obelisk including a single QW at the pyramidal tip (f) and at the column part (g). (h) Bottom-view TEM image showing an InGaN QW in a hexagonal nano-obelisk. (i) Schematic of a nano-obelisk including a single and ultrathin InGaN QW at the side facets and a small island (QD) at the top. All TEM images (a-c,e,f,h) are measured in a bright-field condition except (g) (HAADF STEM).

Single QD spectroscopy. Optical properties of single InGaN QDs embedded in nano-obelisks were examined with various approaches and show several distinctive characteristics compared to conventional InGaN QDs embedded in a planar structure. For the optical characterization of quantum structures in a nano-obelisk, we performed micro-photoluminescence ( $\mu$-PL) experiments using a $325 \mathrm{~nm}$ He-Cd continuous-wave laser and a $345 \mathrm{~nm}$ frequency doubled Ti:sapphire femto-second pulsed laser (Fig. 3a and see Methods). To study a single nano-obelisk, the nano-obelisks dispersed on a patterned Si wafer. As-formed nano-obelisks on the sapphire substrate without dispersing were also studied at the top of the nano-obelisks for analysis of polarization and multi-exciton complexes. Figure $3 \mathrm{~b}$ shows the $\mu$-PL spectrum for a single nanoobelisk at $6 \mathrm{~K}$. Together with a broad InGaN QW emission, a narrow and strong signal was observed at $3.14 \mathrm{eV}$, which indicates atomiclike transitions from the three-dimensionally quantum confined QDs in the nano-obelisk. The full-width at half-maximum (FWHM) of the QD peak was about $500 \mu \mathrm{eV}(0.06 \mathrm{~nm})$, which is close to the instrumental resolution. The linearly polarized emission from QDs is a very important factor for quantum information processing since quantum information could be encoded into the polarization on the single photon qubits, enabling linear optical quantum computing ${ }^{37}$. In Fig. $3 c$, the polarization was analyzed at the top of a single nano-obelisk without being dispersed, and the narrow single QD emission shows a strong linear polarization with a polarization ratio $(P)$ of 0.9 , which is defined as $P=\left(I_{\max }-I_{\min }\right) /$ $\left(I_{\max }+I_{\min }\right)$. Generally, wurtzite crystal structure does not possess in-plane linear polarization along the $c$-plane due to hexagonal symmetry and isotropic biaxial in-plane strain. However, with a small anisotropy in biaxial strain or shape of QDs, electronic structures of valence bands can be deformed, causing in-plane linear polarization ${ }^{38,39}$. The direction of linear polarization can be uniaxial $^{40}$, biaxial ${ }^{39}$, or random ${ }^{41}$. In our case, single QD emissions are strongly polarized in $c$-plane, and the direction of polarization does not follow specific crystal axes. Although the origin of the random polarization direction of QDs is not clear yet, we expect that the strong linear polarization in single QD emission is related to the mixing of $\mathrm{A}$ and $\mathrm{B}$ valance bands due to the anisotropy of shape or strain of QDs ${ }^{41}$.

The formation of multi-exciton complexes was also observed by varying the excitation power. Figure $3 \mathrm{~d}$ shows two narrow peaks located at around $3.21 \mathrm{eV}$ at low excitation power. These two peaks follow the same polarization direction, and exhibit linear dependence of the PL intensity on the excitation power. Hence, they are attributed to the neutral exciton (X) and charged exciton (CX) transitions, respectively, in the same QD. At high excitation power, the two new peaks appear at the lower energy side at around $3.20 \mathrm{eV}$, and these peaks display quadratic dependence of the PL intensity on the excitation power, suggesting that the two peaks are biexciton (XX) and charged biexciton (CXX), respectively. The biexciton binding energy $\left(E_{b}^{X X}\right)$ is described as $E_{b}^{X X}=2 J_{\mathrm{e}-\mathrm{h}}-J_{\mathrm{e}-\mathrm{e}}-J_{\mathrm{h}-\mathrm{h}}$, where $J_{\mathrm{e}-\mathrm{h}}$ is the attractive interaction between electrons and holes and $J_{\mathrm{e}-\mathrm{e}}\left(J_{\mathrm{h}-\mathrm{h}}\right)$ is the repulsive interaction between electrons (holes), respectively ${ }^{42}$. Therefore, $E_{b}^{X X}$ is determined by the spatial distribution of electron and hole wavefunctions, which can have positive or negative signs depending on the QD size. However, most nitride-based QDs have a negative $E_{b}^{X X}$ even with small size QDs because a large built-in electric field separates electron and hole wavefuntions at the top and bottom of the QDs, respectively ${ }^{42}$. Interestingly, the InGaN QDs in the nano-obelisks show an uncommon positive $E_{b}^{X X}$ about $7 \mathrm{meV}$, which is a strong indication of the small size of QDs and a reduced built-in field in the nano-obelisks ${ }^{43}$. We examine this further in the following parts.

Large exciton binding energy in nitride semiconductors enables high temperature operation of excitons, However, for the conventional self-assembled QDs embedded in a planar substrate with a large density of dislocations about $10^{8} \sim 10^{10} / \mathrm{cm}^{2}$ and a huge built-in electric field, exctions in QDs could be easily dissipated and captured by nonradiative centers with increasing temperature. In the case of the single QD embedded in the nano-obelisks, the QD 

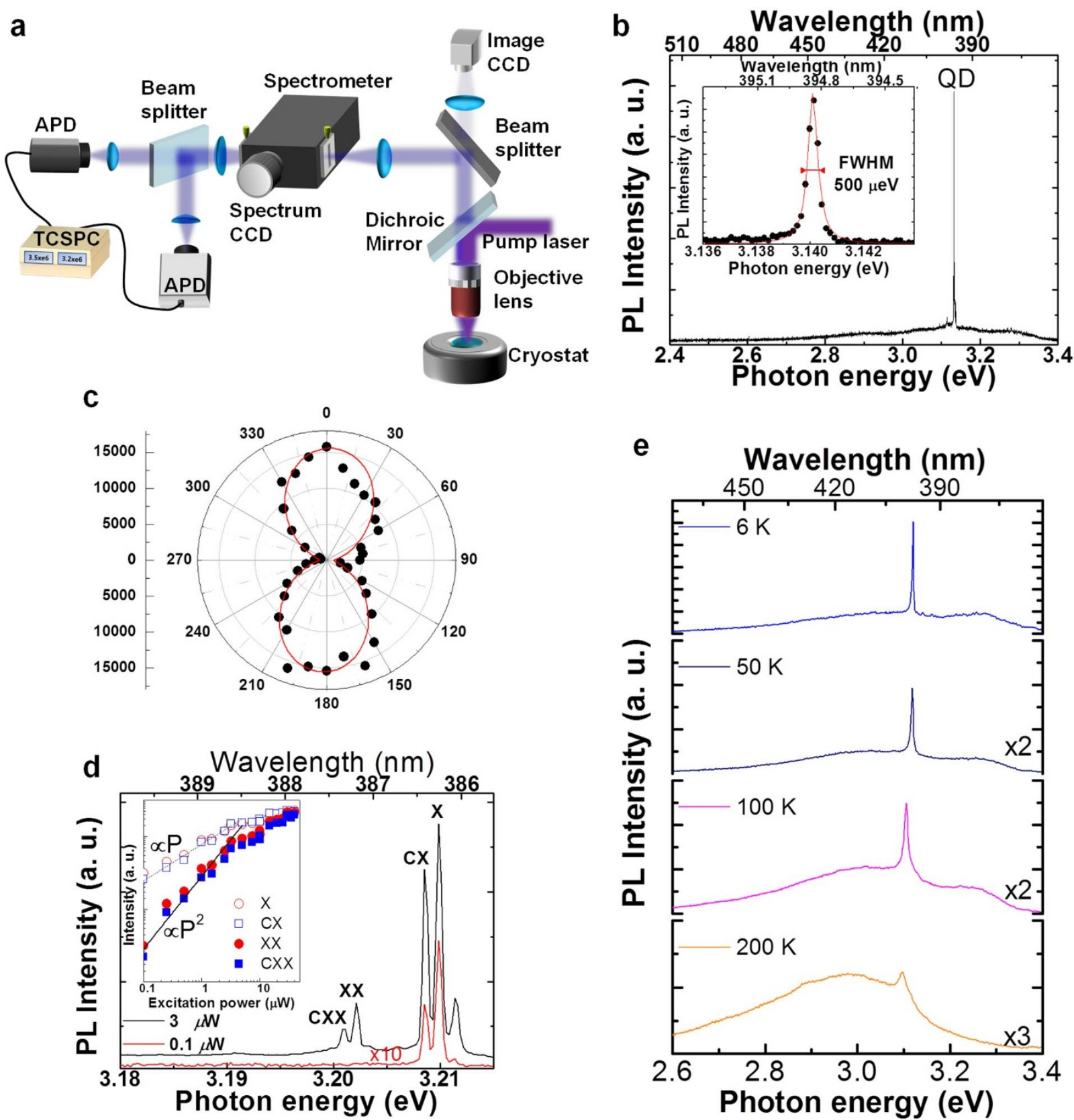

Figure 3 Optical study for a single QD in a nano-obelisk. (a) Schematic of single QD spectroscopy set up. (b) $\mu$-PL spectrum from a single nano-obelisk showing a sharp single InGaN QD emission together with an InGaN QW emission. Inset presents a high-resolution PL spectrum for single QD emission and the red line is a fitted Lorentz curve. (c) Polar plot of the PL intensity of a single QD emission with the polarization direction $(\theta) . \theta=0^{\circ}$ is an arbitrary direction. (d) Formation of mtulti-exiton complexes with excitation power. The single QD shows X and CX transitions at $0.1 \mu \mathrm{W}$, and XX and CXX peaks are also observed at $3 \mu \mathrm{W}$. Inset shows the increase of the PL intensity with the excitation power for X, CX, XX, and CXX transitions. Dashed and solid lines represent linear and quadratic lines, respectively. (e) A Single QD spectrum was measured at various temperatures and the single QD emission can be observed up to $200 \mathrm{~K}$ with an increase of the FWHM from $0.7 \mathrm{meV}$ (6 K) to $12 \mathrm{meV}(200 \mathrm{~K})$.

is spatially well isolated from dislocations. In addition, excitons recombine rapidly, and nano-obelisks extract photons efficiently. From the above advantages, single QD emission could be observed up to $200 \mathrm{~K}$, as shown in Fig. 3e. Beyond $200 \mathrm{~K}$, it was difficult to distinguish single QD emission due to the superimposed broad QW emission. The FWHM of the single QD emission increases with temperature from $0.7 \mathrm{meV}(6 \mathrm{~K})$ to $12 \mathrm{meV}(200 \mathrm{~K})$ due to exciton-phonon interactions ${ }^{24}$.

Carrier recombination dynamics of quantum structures in a nano-obelisk and a planar structure. A significant change in optical properties between the quantum structures in the nano-obelisks and the conventional planar structures occurs in the recombination dynamics. Figure $4 \mathrm{a}$ shows time-resolved $\mu$-PL results for single InGaN QD and InGaN QW emissions in the same single nanoobelisk at $6 \mathrm{~K}$. A single InGaN QW on a planar template grown under the same conditions as the nano-obelisk sample was also compared as a reference. As shown in Fig. 4a, the InGaN QW embedded in a planar structure shows recombination time of $1.1 \mathrm{~ns}$, while the single QD and QW in the nano-obelisk shows remarkably fast recombination of 166 ps and 105 ps, respectively. It is well known that InGaN QWs and QDs embedded in a planar structure have a recombination time on the order of nanoseconds ${ }^{44}$. The main reason for the long recombination time is the existence of 

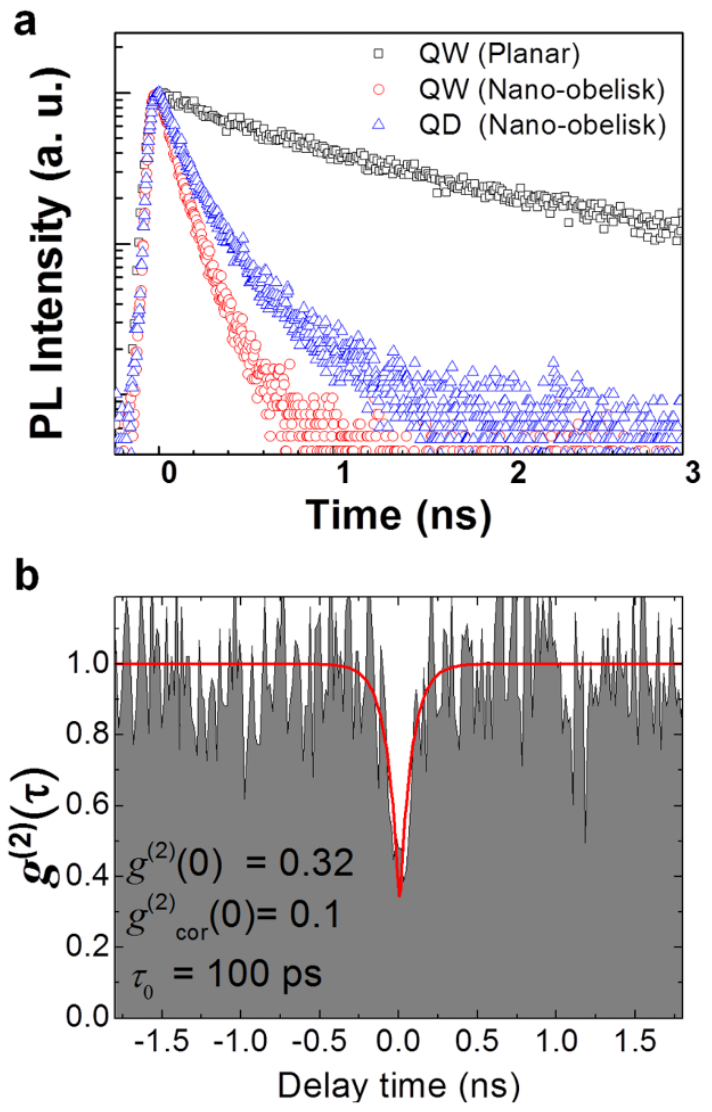

Figure 4 | Ultrafast single photon generation from a single QD in a nanoobelisk. (a) Comparison of recombination curves for QD and QW emissions in the single nano-obelisk, and QW emission in the planar structure. (b) A second order photon correlation result from a single InGaN QD showing an anti-bunching signal. The red line is a fitting curve.

strong spontaneous and piezoelectric polarization fields in the IIInitride system. The built-in electric fields separate the electron and hole wavefunctions (i.e., quantum confined-Stark effect (QCSE)) and inhibit their radiative recombination process, thus limiting the quantum efficiency in the group III-nitride material system ${ }^{45}$. However, the formation of nanostructures could reduce the builtin electric field effectively through strain minimization ${ }^{31,33}$. The reduced built-in electric field and improved spatial coherence in the photonic nanostructures lead to a large increase of the oscillator strength of the quantum structures in the nano-obelisks, which is inversely proportional to the radiative recombination time. QCSE is affected by the amount of the built-in electric field and the size of quantum structures. To determine the dominant factor responsible for a reduced QCSE in the nano-obelisks, we measured the recombination time for thick quantum structures in the nanoobelisks, and fast recombination was also observed even for these structures (Supplementary Section V). Therefore, we conclude that the built-in electric field reduction plays an important role in this unusual fast recombination.

Photon correlation experiment. Finally, we demonstrate the quantum nature of photons from a single QD in a nano-obelisk using the Hanbury Brown and Twiss experiment, which measures the second order correlation function of photons: $g^{(2)}(\tau)=<: I(t) I(t$ $+\tau):>/<I(t)>^{2}$. In the case of single photons, due to their antibunched nature, more than one photon cannot be simultaneously detected by two Si-avalanche photodiodes (APDs) after passing through a 50:50 beam splitter, resulting in a dip at $g^{(2)}(\tau=0)$. The QD emission line was spectrally filtered by a monochromator (Fig. 3a and see Methods). The single QD in the nano-obelisk shows efficient single photon generation with a fast radiative recombination rate. Figure $4 \mathrm{~b}$ is an autocorrelation graph for a single QD emission with a continuous-wave excitation condition at $6 \mathrm{~K}$. It displays a strong anti-bunching signal, indicating non-classical light generation (i.e., single photon state). We fit the autocorrelation graph using the following equation: $g^{(2)}(\tau)=1-\left(1-g^{(2)}(0)\right) \exp \left(-|\tau| / \tau_{0}\right)$. The obtained value for $g^{(2)}(0)$ was 0.32 . The nonzero value at $g^{(2)}(0)$ is mostly due to the uncorrelated background light, dark counts, and limited time resolution of APDs. By excluding the background signal using the modified second-correlation function $g^{(2)}(\tau)=1-\rho^{2}(1-$ $g^{(2)}$ cor $\left.(0)\right) \exp \left(-|\tau| / \tau_{0}\right)$, where $\rho=S /(S+B)$ is the ratio of signal $S$ to background signal $B$ counts $^{3}$, we obtained $g^{(2)}$ cor $(0)=0.1$ with $\rho=$ 0.87 . The anti-bunching signal also shows a very narrow dip, which is strongly associated with the fast recombination time of single QDs in nano-obelisks. From the fitting, the time constant $\tau_{0}$ was about 100 ps. $\left(1 / \tau_{0}=G+1 / \tau_{s p}, G\right.$ is the excitation rate and $1 / \tau_{s p}$ is the spontaneous recombination rate $\left.^{46}\right)$. This fast generation of single photons is difficult to achieve with conventional self-assembled QDs or other single photon sources without a microcavity. For instance, the common recombination times are about $7 \mathrm{~ns}$ for InGaN QDs ${ }^{27}, 1$ ns for InAs QDs ${ }^{10}, 12$ ns for defects in diamond ${ }^{46}$, and $20 \mathrm{~ns}$ for CdSe nanocrystals ${ }^{47}$, respectively. The realization of ultrafast and efficient single photon generation is a promising step toward solid-state quantum optical systems.

\section{Discussion}

With wide-bandgap nitride materials and obelisk shaped nanostructures, we obtained ultrafast violet-colored single photon emitter. The nano-obelisk structure could be easily detached and transferred. Therefore, it is possible to transfer the nano-obelisks to the metal substrate to improve further the light extraction efficiency $^{7}$. The nano-obelisks also provide a fundamental building block for on-chip quantum photonic circuits by integrating with waveguides and detectors. In this study, the spectrum of the broad background QW emission in nano-obelisk was superimposed with the narrow single QD emission, which limits the $g^{(2)}(0)$ value and makes the single QD emission is indistinguishable from the QW emission at high temperature. However, it might be possible to separate the QW and QD emission further by changing the thickness and indium composition of InGaN layer. It is also important to verify whether this fast recombination is dominated by a radiative process or a nonradiative process (e.g., surface recombination). To confirm this, we performed temperature-dependent time-resolved PL experiments. In this experiment, the nano-obelisks maintained their PL intensity and recombination lifetime almost unchanged up to $100 \mathrm{~K}$, and then both decreased after $100 \mathrm{~K}$ (Supplementary Section VI). This is unambiguous evidence that the nonradiative process is suppressed at low temperature below $100 \mathrm{~K}$, and the observed fast recombination time at low temperature is therefore dominated by a fast radiative recombination process rather than a nonradiative process.

In summary, we have demonstrated and investigated ultrafast violet-colored single photon emitting quantum structures embedded in nano-obelisks. The QD embedded in a nano-obelisk offers distinctive advantages over the conventional self-assembled QDs on a planar substrate. A well-isolated single QD could be obtained without a patterning step, and together with increased light extraction efficiency, a significantly reduced built-in electric field was observed in the nano-obelisk structure. This results in peculiar optical properties such as a narrow emission, strong linear polarization, positive biexciton binding energy, and fast recombination for the single InGaN QDs, making efficient and ultrafast single photon generation possible. The fast generation and efficient extraction of a single photon are key elements for realizing a deterministic single photon source. The formation of quantum structures in the nano-obelisk 
overcomes many limitations in conventional self-assembled QDs in the planar structure and shows strong potential as a practical single photon source with nitride-based single QDs.

\section{Methods}

Sample preparations and structural characterization. $2 \mu \mathrm{m}$-thick $\mathrm{GaN}$ wafers were prepared using MOCVD on a $c$-plane sapphire substrate, and then GaN wafers were etched by a chemical vapor-phase etching method with the following etching conditions: temperature of $1000^{\circ} \mathrm{C}$, pressure of 771 Torr, etching gas of $1000 \mathrm{sccm}$ of $\mathrm{HCl}$ and $250 \mathrm{sccm}$ of $\mathrm{NH}_{3}$ during $5 \mathrm{~min}$. After etching, we grew a InGaN active layer and a $\mathrm{GaN}$ capping layer on the etched $\mathrm{GaN}$ nanostructure templates and $c$-plane $\mathrm{GaN}$ templates using MOCVD at the same growth conditions with growth temperature of $680^{\circ} \mathrm{C}$ for InGaN active layers. The indium composition was $20 \%$ for the InGaN QW in the planar structure, and the $5 \mathrm{~nm}$-thick QW in the nano-obelisks shows a similar value, but it was difficult to ascertain the exact indium composition for an ultrathin InGaN QW in nano-obelisks. Structural characteristics of the samples were investigated using SEM (Hitachi S-4800) and TEM (JEM-ARM200F). Crosssectional TEM samples were prepared by mechanical polishing and an ion-milling process (Gatan PIPS691)

Single QD spectroscopy. For the single QD spectroscopy, the sample was mounted on a cold stage in a low-vibration cryostat (Advanced Research System), and a $325 \mathrm{~nm}$ He-Cd laser and a $345 \mathrm{~nm}$ mode-locked Ti:sapphire laser with a repetition rate of $80 \mathrm{MHz}$ were used as continuous-wave and pulsed excitation sources, respectively. The laser was focused by a $100 \times($ N.A. $=0.5$, Mitutoyo) objective lens and the sample emission was collected by the same objective lens and sent to a spectrometer ( $0.75 \mathrm{~m}$ focal length, Princeton Instruments) coupled with an thermoelectric cooled CCD array detector to measure the emission wavelength and intensity. For $\mu$-PL experiments, we separated the nano-obelisks by a knife and dispersed them on a Si wafer. However, for polarization dependent $\mu$-PL measurement, an as-formed nano-obelisk sample was used without being dispersed and the polarization of the QD emission was hence analyzed at the top of the sample. The linear polarization of the PL intensity was analyzed by rotating a $\lambda / 2$ wave plate in front of a linear polarizer. To obtain photon correlation data from a single QD, we used two APDs (temporal resolution of $\sim 40$ ps, ID Quantique) after a 50:50 beam splitter, and the APD signals were processed by a time-correlated single-photon counting system (Picoharp300, Picoquant). The single QD emission was not observed from every single nanostructure but rather a small percent of the nanostructures. The background signal $B$ in $g^{(2)}(\tau)$ was simply measured by tuning the detection wavelength to the region outside of the QD emission, which includes a dark count in the APD and un uncorrelated emission from the QW at around $400 \mathrm{~nm}$.

1. Alber, G. et al. Quantum information. (Springer, Berlin, 2001)

2. Pelton, M. et al. Efficient source of single photons: a single quantum dot in a micropost microcavity. Phys. Rev. Lett. 89, 233602 (2002).

3. Heindel, T. et al. Electrically driven quantum dot-micropillar single photon source with 34\% overall efficiency. Appl. Phys. Lett. 96, 011107 (2010).

4. Shields, A. J. Semiconductor quantum light sources. Nat. Photon. 1, 215-223 (2007).

5. Hennessy, K. et al. Quantum nature of a strongly coupled single quantum dotcavity system. Nature 445, 896-899 (2007)

6. Babinec, T. M. et al. A diamond nanowire single-photon source. Nat. Nanotech. $\mathbf{5}$, 195-199 (2010).

7. Reimer, M. E. et al. Bright single-photon sources in bottom-up tailored nanowires Nat. Commun. 3, 737 (2012)

8. Bleuse, J. et al. Inhibition, enhancement, and control of spontaneous emission in photonic nanowires. Phys. Rev. Lett. 106, 103601 (2011).

9. Claudon, J. et al. A highly efficient single-photon source based on a quantum dot in a photonic nanowire. Nat. Photon. 4, 174-177 (2010)

10. Thompson, R. M. et al. Single-photon emission from exciton complexes in individual quantum dots. Phys. Rev. B 64, 201302 (2001).

11. Santori, C., Pelton, M., Solomon, G., Dale, Y. \& Yamamoto, Y. Triggered single photons from a quantum dot. Phys. Rev. Lett. 86, 1502-1505 (2001).

12. Bounouar, S. et al. Ultrafast room temperature single-photon source from nanowire-quantum Dots. Nano Lett. 12, 2977-2981 (2012).

13. Rarity, J. G., Tapster, P. R. \& Gorman, P. M. Secure free-space key exchange to $1.9 \mathrm{~km}$ and beyond. J. Mod. Opt. 48, 1887-1901 (2001).

14. Wu, Y., Payne, M. G., Hagley, E. W. \& Deng, L. Ultraviolet single-photons on demand and entanglement of photons with a large frequency difference. Phys. Rev. A 70, 063812 (2004)

15. Hadfield, R. H. Single-photon detectors for optical quantum information applications. Nat Photon 3, 696-705 (2009).

16. Zaske, S. et al. Visible-to-telecom quantum frequency conversion of light from a single quantum emitter. Phys. Rev. Lett. 109, 147404 (2012).

17. Ramvall, P., Tanaka, S., Nomura, S., Riblet, P. \& Aoyagi, Y. Observation of confinement-dependent exciton binding energy of GaN quantum dots. Appl. Phys. Lett. 73, 1104-1106 (1998).

18. Aleksiejunas, R. et al. Determination of free carrier bipolar diffusion coefficient and surface recombination velocity of undoped GaN epilayers. Appl. Phys. Lett. 83, 1157-1159 (2003).
19. Schubert, E. F., Gessmann, T. \& Kim, J. K. Light emitting diodes. (Wiley Online Library, 2005).

20. Kako, S. et al. A gallium nitride single-photon source operating at $200 \mathrm{~K}$. Nat. Mater. 5, 887-892 (2006)

21. Kim, J. H. et al. Carrier transfer and recombination dynamics of a long-lived and visible range emission from multi-stacked GaN/AlGaN quantum dots. Appl. Phys. Lett. 97, 061905 (2010).

22. Vartuli, C. B. et al. Wet chemical etching survey of III-nitrides. Solid-State Electron. 41, 1947-1951 (1997)

23. Hahn, Y. B. et al. High-density plasma-induced etch damage of InGaN/GaN multiple quantum well light-emitting diodes. J. Appl. Phys. 92, 1189-1194 (2002).

24. Ostapenko, I. A. et al. Exciton acoustic-phonon coupling in single GaN/AIN quantum dots. Phys. Rev. B 85, 081303 (2012).

25. Simon, J. et al. Direct comparison of recombination dynamics in cubic and hexagonal GaN/AlN quantum dots. Phys. Rev. B 68, 035312 (2003).

26. Adelmann, C. et al. Self-assembled InGaN quantum dots grown by molecularbeam epitaxy. Appl. Phys. Lett. 76, 1570-1572 (2000).

27. Jarjour, A. F. et al. Cavity-enhanced blue single-photon emission from a single InGaN/GaN quantum dot. Appl. Phys. Lett. 91, 052101 (2007).

28. Kremling, S. et al. Single photon emission from InGaN/GaN quantum dots up to 50 K. Appl. Phys. Lett. 100, 061115 (2012)

29. Deshpande, S., Heo, J., Das, A. \& Bhattacharya, P. Electrically driven polarized single-photon emission from an InGaN quantum dot in a GaN nanowire. Nat. Commun. 4, 1675 (2013).

30. Kim, J. H. et al. Dislocation-eliminating chemical control method for high efficiency GaN-based light emitting nanostructures. Cryst. Growth Des. 12, 1292-1298 (2012).

31. Chang, H. et al. Strong luminescence from strain relaxed $\mathrm{InGaN} / \mathrm{GaN}$ nanotips for highly efficient light emitters. Opt. Express 15, 9357-9365 (2007)

32. Songmuang, R. et al. Strong suppression of internal electric field in GaN/AlGaN multi-layer quantum dots in nanowires. Appl. Phys. Lett. 99, 141914 (2011).

33. Liang, Z. et al. Built-in electric field minimization in (In, Ga) N nanoheterostructures. Nano Lett. 11, 4515-4519 (2011).

34. Ko, Y.-H. et al. Electrically Driven Quantum Dot/Wire/Well Hybrid LightEmitting Diodes. Adv. Mater. 23, 5364-5369 (2011)

35. Edwards, P. R. et al. Quantum dot emission from site-controlled InGaN/GaN micropyramid arrays. Appl. Phys. Lett. 85, 4281-4283 (2004).

36. Hartmann, A., Ducommun, Y., Loubies, L., Leifer, K. \& Kapon, E. Structure and photoluminescence of single AlGaAs/GaAs quantum dots grown in inverted tetrahedral pyramids. Appl. Phys. Lett. 73, 2322-2324 (1998).

37. Kok, P. et al. Linear optical quantum computing with photonic qubits. Rev. Mod Phys. 79, 135-174 (2007).

38. Fu, D. et al. Modification of the valence band structures of polar and nonpolar plane wurtzite-GaN by anisotropic strain. J. Appl. Phys. 106, 023714-023718 (2009).

39. Winkelnkemper, M. et al. Polarized emission lines from A- and B-type excitonic complexes in single InGaN/GaN quantum dots. J. Appl. Phys. 101, 113708-113704 (2007)

40. Hsu, C.-W. et al. Single Excitons in InGaN Quantum Dots on GaN Pyramid Arrays. Nano Lett. 11, 2415-2418 (2011).

41. Bardoux, R. et al. Polarized emission from GaN/AlN quantum dots: Single-dot spectroscopy and symmetry-based theory. Phys. Rev. B 77, 235315 (2008).

42. Williams, D. P., Andreev, A. D. \& O’Reilly, E. P. Self-consistent calculations of exciton, biexciton and charged exciton energies in InGaN/GaN quantum dots. Superlattices and Microstruct. 36, 791-798 (2004).

43. Tomić, S. \& Vukmirović, N. Excitonic and biexcitonic properties of single GaN quantum dots modeled by 8 -band $\mathrm{k} \cdot \mathrm{p}$ theory and configuration-interaction method. Phys. Rev. B 79, 245330 (2009)

44. Robinson, J. W. et al. Time-resolved dynamics in single InGaN quantum dots. Appl. Phys. Lett. 83, 2674-2676 (2003).

45. Ryou, J.-H. et al. Control of quantum-confined stark effect in InGaN-based quantum wells. IEEE J. Sel. Topics Quantum Electron. 15, 1080-1091 (2009).

46. Kurtsiefer, C., Mayer, S., Zarda, P. \& Weinfurter, H. Stable solid-state source of single photons. Phys. Rev. Lett. 85, 290-293 (2000).

47. Brokmann, X. et al. Colloidal CdSe/ZnS quantum dots as single-photon sources. New J. Phys. 6, 99 (2004).

\section{Acknowledgments}

This work was supported by the National Research Foundation

(NRF-2013R1A2A1A01016914), the WCU Program (No. R31-2008-000-1071-0) of the Ministry of Education, the KAIST EEWS Initiative, and the GRC project of KI for the NanoCentury.

\section{Author contributions}

J.H.K. and Y.H.C. designed all experiments. Y.H.K. and S.M.K. served the GaN epilayer samples and assisted with regrowth process using MOCVD. J.H.K. performed the chemical etching process and carried out the structure properties and optical properties of samples using SEM, TEM, time integrated PL, and time resolved PL. J.H.K. along with S.H.G. 
performed singe QD spectroscopy using $\mu$-PL and HBT set up. J.H.K. and Y.H.C. analyzed data and wrote the manuscript. All authors have reviewed and discussed the results and conclusions of this manuscript.

\section{Additional information}

Supplementary information accompanies this paper at http://www.nature.com/ scientificreports

Competing financial interests: The authors declare no competing financial interests.

How to cite this article: Kim, J.-H., Ko, Y.-H., Gong, S.-H., Ko, S.-M. \& Cho, Y.-H. Ultrafast single photon emitting quantum photonic structures based on a nano-obelisk. Sci. Rep. 3, 2150; DOI:10.1038/srep02150 (2013). (i) (\$) This work is licensed under a Creative Commons AttributionNonCommercial-NoDerivs 3.0 Unported license. To view a copy of this license, visit http://creativecommons.org/licenses/by-nc-nd/3.0 